\title{
Distributed Collision-Free Motion Coordination on a Sphere: A Conic Control Barrier Function Approach
}

\author{
Tatsuya Ibuki ${ }^{1}$, Sean Wilson ${ }^{2}$, Aaron D. Ames ${ }^{3}$, and Magnus Egerstedt ${ }^{2}$
}

\begin{abstract}
This paper studies a distributed collision avoidance control problem for a group of rigid bodies on a sphere. A rigid body network, consisting of multiple rigid bodies constrained to a spherical surface and an interconnection topology, is first formulated. In this formulation, it is shown that motion coordination on a sphere is equivalent to attitude coordination on the 3-dimensional Special Orthogonal group. Then, an angle based control barrier function that can handle a geodesic distance constraint on a spherical surface is presented. The proposed control barrier function is then extended to a relative motion case and applied to a collision avoidance problem for a rigid body network operating on a sphere. Each rigid body chooses its control input by solving a distributed optimization problem to achieve a nominal distributed motion coordination strategy while satisfying constraints for collision avoidance. The proposed collision-free motion coordination law is validated through simulation.
\end{abstract}

\section{INTRODUCTION}

Safe and distributed motion coordination of individual robots within a multi-robot collective is required to solve many tasks, like formation, flocking, and coverage control [1]-[5]. While many studies focused on motion coordination of a multi-robot system consider the 3-dimensional (3D) Euclidean space or a 2-dimensional (2D) plane as a workspace, a spherical surface is also often required [6]-[13]. This motion coordination on a sphere is motivated not only by theoretical interests but also by some industrial application such as planetary-scale motion coordination/localization in the space/ocean, and vehicle coordination on the surface with a large radius of curvature. Moreover, spherical motion constraints can be considered for motion control of single arm manipulators or attitude control of pan-tilt cameras, and recently, the constraints are also utilized in dynamics of cooperative transportation of a payload with multiple flying vehicles [14], [15].

No matter what motion constrained workspace a multirobot system operates in, effective collision avoidance is an essential requirement to guarantee hardware safety. A potential function based approach is one common collision avoidance strategy for multi-agent systems [11], [16]-[20].

*This work was supported in part by JSPS KAKENHI under Grant 18 K13775 and in part by the US National Science Foundation through Grant 1531195 .

${ }^{1}$ T. Ibuki is with Department of Systems and Control Engineering, School of Engineering, Tokyo Institute of Technology, Tokyo 152-8550, Japan ibukiesc.e.titech.ac.jp

${ }^{2} \mathrm{~S}$. Wilson and M. Egerstedt are with the School of Electrical and Computer Engineering, Georgia Institute of Technology, Atlanta, GA 30332, USA \{sean.t.wilson, magnus\}@gatech.edu

${ }^{3}$ A. D. Ames is with the Department of Mechanical and Civil Engineering, California Institute of Technology, Pasadena, CA 91125, USA ames@caltech.edu
This technique introduces a nonnegative scalar function that increases as a robot approaches obstacles, like other robots or environmental hazards. Then, collision-free motion coordination methods incorporate its negative gradient to guarantee a safe operating distance. However, the potential function often needs to be infinite at the obstacle boundary, which causes overcaution about safety, i.e., less control performance.

More recently, constraint based optimization methods have been used to guarantee robot safety during operation [21], [22]. Here, a scalar function describing a safe set, called a control barrier function $(C B F)$, is introduced, and the forward invariance of the dynamics within the safe set is guaranteed via the constraints derived by the CBF. In this approach, the control input is given by solving an optimization problem to achieve a control task as much as possible while guaranteeing the safety. This technique is also applied to collision-free motion coordination problems for multi-agent systems as in [4], [23], [24]. Most of the existing studies, however, consider collision avoidance problems with standard Euclidean distances, i.e., in 3D space or on a 2D plane. This work extends the CBF based approach to a collision-free motion coordination method on a spherical surface, where the safety is defined with geodesic distances.

This paper first formulates a rigid network consisting of multiple rigid bodies with their motion dynamics constrained to a spherical surface and an interconnection topology. We show that motion coordination on a sphere is equivalent to attitude coordination on the 3D Special Orthogonal group: $S O(3)$. Then, as a bridge to a collision avoidance problem on a sphere, we develop a CBF based safe control technique on $S O(3)$. This approach is first applied to an angle based (conic) constraint satisfaction problem, and by extending it to a relative motion case, we propose a collision-free motion coordination law for a rigid body network on a sphere. In the proposed method, each rigid body selects its control input by solving a distributed optimization problem to achieve a given motion coordination task as much as possible while guaranteeing collision avoidance. The effectiveness of the proposed approach is demonstrated via simulation.

The main contributions of this paper are twofold. First, we develop a new $\mathrm{CBF}$ to handle constraints on $S O(3)$ by extending the classical CBF methods for vector fields presented in [21], [22]. Here, we also provide an example of safe attitude control for a single rigid body with a conic constraint. Second, we extend this kind of CBFs to a relative motion case, and propose a novel distributed collision-free motion coordination method for a rigid body network on a spherical surface. 


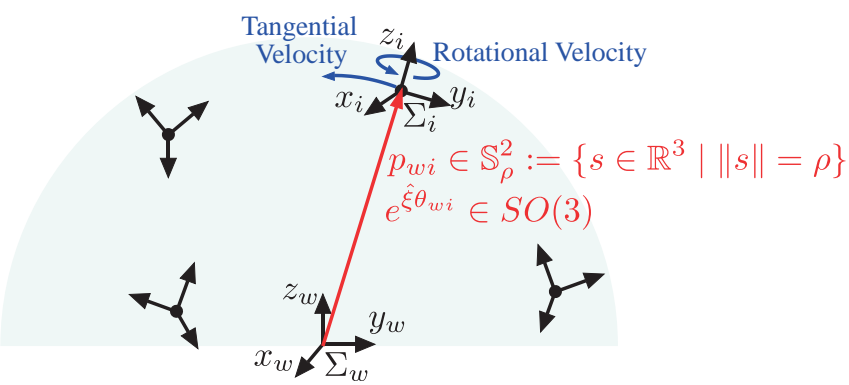

Fig. 1. Multiple rigid bodies on sphere. The origin of the world frame $\Sigma_{w}$ is located at the center of a sphere with radius $\rho$, and the position of each rigid body is constrained on the surface of the sphere.

\section{PROBLEM SETTINGS}

\section{A. Rigid Body Motion}

As a preliminary, the motion dynamics of multiple rigid bodies in general 3D space are first introduced. Let us consider a set of $n$ rigid bodies. Each rigid body $i \in$ $\{1, \ldots, n\}$ has a body fixed frame $\Sigma_{i}$ in a world frame $\Sigma_{w}$. The position and attitude of rigid body $i$ in $\Sigma_{w}$ are represented by $\left(p_{w i}, e^{\hat{\xi}_{w i} \theta_{w i}}\right) \in S E(3)$. Here, $e^{\hat{\xi} \theta} \in S O(3)$ is the exponential coordinate of the rotation matrix with the rotation axis $\xi \in \mathbb{R}^{3}(\|\xi\|=1)$ and angle $\theta \in[-\pi, \pi)$ [25]. The operator $\wedge: \mathbb{R}^{3} \rightarrow s o(3)$ gives $\hat{a} b=a \times b$ for any $3 \mathrm{D}$ vectors $a, b \in \mathbb{R}^{3}$, and $\vee: s o(3) \rightarrow \mathbb{R}^{3}$ is its inverse operator. For the ease of representation, $\hat{\xi}_{w i} \theta_{w i}$ is written as $\hat{\xi} \theta_{w i}$ throughout this paper.

The body translational and rotational velocity of rigid body $i$ relative to $\Sigma_{w}$ is denoted by $v_{i} \in \mathbb{R}^{3}$ and $\omega_{i} \in$ $\mathbb{R}^{3}$, respectively. Then, for each rigid body $i$, we have the following rigid body motion [25]:

$$
\dot{p}_{w i}=e^{\hat{\xi} \theta_{w i}} v_{i}, \quad e^{\hat{\xi} \theta_{w i}}=e^{\hat{\xi} \theta_{w i}} \hat{\omega}_{i} .
$$

\section{B. Rigid Body Motion on a Sphere}

Let us next consider the motion dynamics of multiple rigid bodies constrained to the surface of a sphere with radius $\rho>0$. Without loss of generality, let the origin of the world frame $\Sigma_{w}$ be located at the center of the sphere, and the direction of the $z$-axis of each body frame $\Sigma_{i}, i \in\{1, \ldots, n\}$ coincide with the radial direction (see Fig. 1). In this case, each rigid body $i$ has the spherical constraint with the basis axis $e_{3}:=\left[\begin{array}{lll}0 & 0 & 1\end{array}\right]^{\mathrm{T}} \in \mathbb{S}^{2}:=\left\{s \in \mathbb{R}^{3} \mid\|s\|=1\right\}$ as

$$
p_{w i}=\rho e^{\hat{\xi} \theta_{w i}} e_{3} \text {. }
$$

Substituting (2) into (1), we obtain the following rigid body motion on a sphere for each rigid body $i$ :

$$
\begin{aligned}
& \dot{p}_{w i}=-\rho e^{\hat{\xi} \theta_{w i}} \hat{e}_{3} \omega_{i} \\
& \dot{e}^{\hat{\xi} \theta_{w i}}=e^{\hat{\xi} \theta_{w i}} \omega_{i} .
\end{aligned}
$$

Remark 1 Under the spherical constraint (2), the position of each rigid body $i$ is determined by its attitude $e^{\hat{\xi} \theta_{w i}}$. Compared with the rigid body motion (1), the translational body velocity $v_{i}$ is also determined by the rotational body velocity $\omega_{i}$, i.e., $v_{i}=-\rho \hat{e}_{3} \omega_{i}$. Therefore, the freedom of motion of each rigid body is 3 , which is analogous to the $2 \mathrm{D}$ vehicle case on a plane 1 (2D position and 1D attitude).

From the observation in Remark 1] motion coordination on a sphere, like formation and collision avoidance, is equivalent to attitude coordination on $S O(3)$. Therefore, for the convenience of introducing control barrier functions in the subsequent discussion, this paper focuses on attitude control on $S O(3)$ and considers the rotational body velocity $\omega_{i}$ as the control input of each rigid body $i$. The actual control input on the sphere is then given by the first two elements of $v_{i}=-\rho \hat{e}_{3} \omega_{i}=\left[\rho \omega_{i, y}-\rho \omega_{i, x} 0\right]^{\mathrm{T}}$ and the third element of $\omega_{i}$ (i.e., $\omega_{i, z}$ ) for the notation $\omega_{i}=\left[\begin{array}{lll}\omega_{i, x} & \omega_{i, y} \omega_{i, z}\end{array}\right]^{\mathrm{T}}$.

\section{Rigid Body Network on a Sphere and Research Objective}

In this paper, we suppose that a motion coordination strategy to achieve a control task is given a priori, and mainly focus on a distributed collision avoidance problem. Here, the interconnection topology between rigid body pairs for the given motion coordination strategy is represented by a directed graph $\mathcal{G}=(\mathcal{V}, \mathcal{E})$, composed of the rigid body set $\mathcal{V}:=\{1, \ldots, n\}$ and the edge set $\mathcal{E} \subset \mathcal{V} \times \mathcal{V}$. We also define the neighbor set of each rigid body $i$ for the strategy as $\mathcal{N}_{i}:=\{j \in \mathcal{V} \mid(j, i) \in \mathcal{E}\}$. Then, $j \in \mathcal{N}_{i}$ means that rigid body $i$ obtains information about rigid body $j$.

Throughout this work, a group of $n$ rigid bodies with the rigid body motion on a sphere (3) and the interconnection topology $\mathcal{G}$ is called a rigid body network on a sphere. In this formulation, the main objective of this work is to develop a distributed collision avoidance method for a rigid body network on a sphere.

\section{CONIC CONTROL BARRIER FUNCTIONS}

As a bridge to a collision avoidance problem for a rigid body network on a sphere, this section presents a geometric control barrier function $(\mathrm{CBF})$ on $S O(3)$ (refer to [21] for more details about CBFs). We note that only a single rigid body $i$ with the attitude dynamics described in $3 \mathrm{~b}$ is considered in this section.

\section{A. Control Barrier Functions on $S O(3)$}

Consider the attitude dynamics (3b), for rigid body $i$ in the world frame $\Sigma_{w}$, with $e^{\hat{\xi} \theta_{w i}} \in \mathcal{S} \subset S O(3), \omega_{i} \in \Omega \subset \mathbb{R}^{3}$, and the constraint set $C_{o}$ defined as

$$
C_{o}:=\left\{e^{\hat{\xi} \theta_{w i}} \in S O(3) \mid h\left(e^{\hat{\xi} \theta w i}\right) \geq 0\right\} .
$$

Here, $h: S O(3) \rightarrow \mathbb{R}$ is a continuously differentiable function. Then, we provide a $\mathrm{CBF}$ definition on $S O(3)$ similarly to [21] as follows:

Definition 1 The function $h\left(e^{\hat{\xi} \theta_{w i}}\right)$ is called a zeroing $C B F$ $(Z C B F)$ defined on the set $\mathcal{S}$ with $C_{o} \subseteq \mathcal{S} \subset S O(3)$, if there exists an extended class $\mathcal{K}$ function $\alpha: \mathbb{R} \rightarrow \mathbb{R}$ satisfying

$$
\sup _{\omega_{i} \in \Omega}\left(\dot{h}\left(e^{\hat{\xi} \theta_{w i}}\right)+\alpha\left(h\left(e^{\hat{\xi} \theta_{w i}}\right)\right)\right) \geq 0 \forall e^{\hat{\xi} \theta_{w i}} \in \mathcal{S} .
$$

${ }^{1}$ A plane can be interpreted as the special case of the spherical surface with radius $\rho=\infty$. 


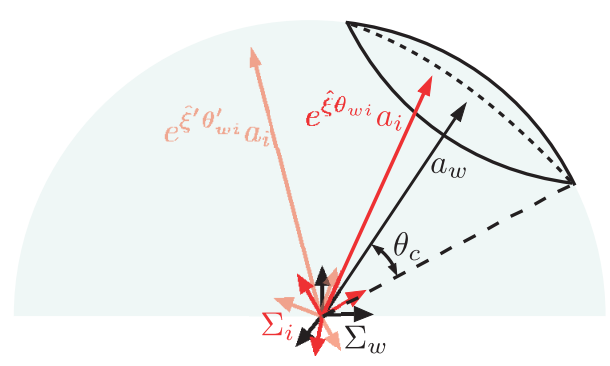

Fig. 2. Conic constraints. Constraint 5a: The basis axis $e^{\hat{\xi} \theta} w^{i} a_{i}$ is constrained inside and on the boundary of the conic region determined by $a_{w}$ and $\theta_{c}$ in $\Sigma_{w}$. Constraint $\left[5 \mathrm{~b}\right.$ : The basis axis $e^{\hat{\xi}^{\prime} \theta_{w i}^{\prime} a_{i}}$ is constrained outside and on the boundary of the conic region (shown by the semitransparent illustration).

Notice in Definition 1 that the control input $\omega_{i}$ appears in $\dot{h}\left(e^{\hat{\xi} \theta_{w i}}\right)$ from $(3 \mathrm{~b})$. Then, we have the following theorem:

Theorem 1 If the function $h\left(e^{\hat{\xi} \theta_{w i}}\right)$ is a ZCBF on $\mathcal{S}$, then any Lipschitz continuous controller $\omega_{i}: \mathcal{S} \rightarrow \Omega$ satisfying $\dot{h}\left(e^{\hat{\xi} \theta_{w i}}\right)+\alpha\left(h\left(e^{\hat{\xi} \theta_{w i}}\right)\right) \geq 0$ will render the set $C_{o}$ forward invariant.

Proof: By appropriately considering the subspace $\mathcal{S} \subset$ $S O(3)$, the attitude dynamics $3 \mathrm{~b}$ can be rewritten by local Lipschitz continuous dynamics in vector form (see Appendix). Corollary 2 in [21] can be thus applied.

Theorem 1 means that the attitude $e^{\hat{\xi} \theta_{w i}}$ remains in the set $C_{o}$ for all time.

\section{B. Definitions of Conic Control Barrier Functions}

Let us now provide explicit definitions of $h\left(e^{\hat{\xi} \theta_{w i}}\right)$ to represent cone-type (conic) attitude constraints used in this work, which are motivated by [29]-[31]. Let $a_{w}, a_{i} \in \mathbb{S}^{2}$ be the basis axes in the world frame $\Sigma_{w}$ and the body frame $\Sigma_{i}$, respectively. Notice then that $e^{\hat{\xi} \theta_{w i}} a_{i}$ means the direction of the basis axis of $\Sigma_{i}$ viewed from $\Sigma_{w}$.

Consider two kinds of inequality constraints as follows:

$$
\begin{aligned}
& a_{w}^{\mathrm{T}} e^{\hat{\xi} \theta_{w i}} a_{i} \geq \cos \theta_{c}, \\
& a_{w}^{\mathrm{T}} e^{\hat{\xi} \theta_{w i}} a_{i} \leq \cos \theta_{c},
\end{aligned}
$$

where $\theta_{c} \in[0, \pi / 2]$ is the constraint parameter to determine the size of the conic region. These constraints are formed by the inner product of the basis axes $a_{w}$ and $e^{\hat{\xi} \theta_{w i}} a_{i}$. The constraint (5a) (constraint (5b) ) thus means that the head of the vector $e^{\hat{\xi} \theta} \theta_{w i} a_{i}$ is constrained inside (outside) and on the boundary of the conic region determined by $a_{w}$ and $\theta_{c}$ in $\Sigma_{w}$ (see Fig. 2). These kinds of constraints are called conic constraints in this paper.

We next develop a ZCBF to guarantee the conic constraint (5a). Based on (5a), an angle based $\mathrm{ZCBF}$, referred to as a conic $C B F$ in this work, is defined as $h\left(e^{\hat{\xi} \theta_{w i}}\right):=$ $a_{w}^{\mathrm{T}} e^{\hat{\xi} \theta_{w i}} a_{i}-\cos \theta_{c}$. This enables us to represent the attitude set satisfying the conic constraint (5a) by (4).

Then, we have the following corollary from Theorem 1

Corollary 1 Any Lipschitz continuous controller $\omega_{i}: \mathcal{S} \rightarrow$ $\Omega$ satisfying

$$
-a_{w}^{\mathrm{T}} e^{\hat{\xi} \theta_{w i}} \hat{a}_{i} \omega_{i}+\alpha\left(a_{w}^{\mathrm{T}} e^{\hat{\xi} \theta_{w i}} a_{i}-\cos \theta_{c}\right) \geq 0
$$

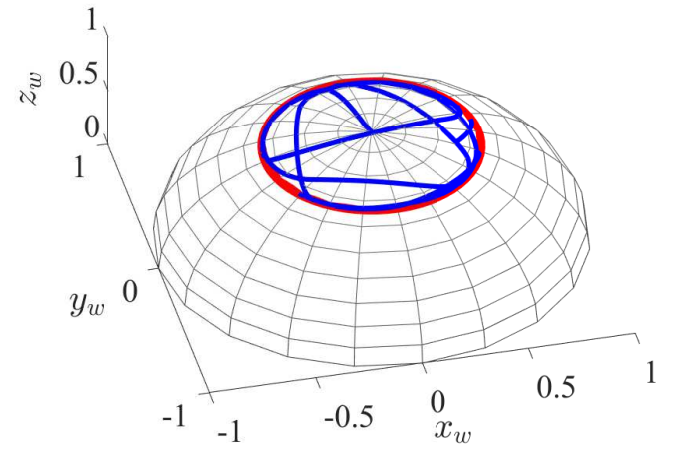

Fig. 3. Time trajectory of attitude. The blue line shows the time trajectory of the head of the vector $e^{\hat{\xi} \theta_{w i}} a_{i}$ in $\Sigma_{w}$. The attitude is constrained inside or on the boundary of the conic constraint 5a represented by the red line.

will render the set $C_{o}$ forward invariant. 2

The following corollary also holds for the constraint $(5 \mathrm{~b})$ :

Corollary 2 Any Lipschitz continuous controller $\omega_{i}: \mathcal{S} \rightarrow$ $\Omega$ satisfying

$$
a_{w}^{\mathrm{T}} e^{\hat{\xi} \theta_{w i}} \hat{a}_{i} \omega_{i}-\alpha\left(a_{w}^{\mathrm{T}} e^{\hat{\xi} \theta_{w i}} a_{i}-\cos \theta_{c}\right) \geq 0
$$

will render the set $C_{o}$ with $h\left(e^{\hat{\xi} \theta_{w i}}\right)=-a_{w}^{\mathrm{T}} e^{\hat{\xi} \theta_{w i}} a_{i}+\cos \theta_{c}$ forward invariant.

\section{Safe Control with Conic Control Barrier Functions}

Corollary 1 enables us to propose the attitude control input provided by the solution of the quadratic program to guarantee the conic constraint (5a) as follows:

$$
\omega_{i}^{*}=\arg \min _{\omega_{i} \in \Omega}\left\|\omega_{i}-\omega_{\text {nom,i }}\right\|^{2} \text { s.t. (6) } \text {. }
$$

Here, $\omega_{\text {nom }, i} \in \mathbb{R}^{3}$ is the nominal controller to achieve a given control task. The optimization in (7) implies that rigid body $i$ tries to achieve the given control task as much as possible while guaranteeing the conic constraint (5a).

As verification, we apply the control input (7) to the attitude dynamics (3b) with $a_{w}=a_{i}=e_{3}, \theta_{c}=\pi / 6$, and a geometric trajectory tracking law as the nominal controller $\omega_{\text {nom }, i}$. Here, the desired trajectory is intentionally set so that the conic constraint (5a) is violated if the nominal controller is directly applied. Fig. 3 depicts the time trajectory of the head of the vector $e^{\hat{\xi} \theta_{w i}} a_{i}$ in $\Sigma_{w}$ by the blue line and the boundary of the conic constraint (5a) by the red one. We see from this figure that the conic constraint (5a) is satisfied.

\section{COLLISION-FREE MOTION COORDINATION}

\section{A. Definitions of Collisions on a Sphere}

As stated in Section II-B this paper focuses on attitude control on $S O(3)$ to deal with motion coordination of a rigid body network on a sphere. We first define the relative attitude of rigid body $j$ to rigid body $i$ as $e^{\hat{\xi} \theta_{i j}}:=e^{-\hat{\xi} \theta_{w i}} e^{\hat{\xi} \theta_{w j}} \in$

\footnotetext{
${ }^{2}$ The property $\hat{a} b=-\hat{b} a$ for any $3 \mathrm{D}$ vectors $a, b \in \mathbb{R}^{3}$ is used to obtain the condition 6 .
} 


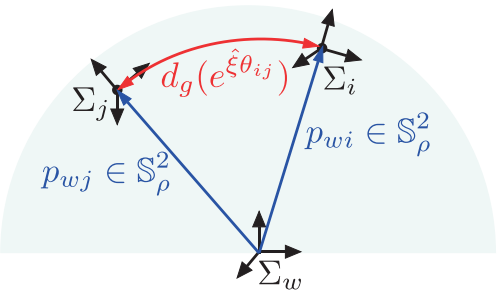

(a) Geodesic distance on sphere.

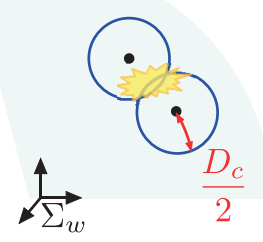

(b) Collision.
Fig. 4. Cartoon illustrations of (a) geodesic distance and (b) collision. (a): The geodesic distance is defined as the arc length of the spherical surface. (b): The collision occurs when the geodesic distance between two rigid bodies is less than their geodesic diameter $D_{c}$.

$S O(3)$. Then, we extend the conic CBF approach presented in Section IIIto a collision avoidance problem by considering the relative attitude case of Corollary 2 with $a_{i}=e_{3} \forall i \in \mathcal{V}$.

Under the spherical constraint (2), the geodesic distance between rigid body $i$ and rigid body $j$ is defined as the arc length of the spherical surface (see Fig. 4(a)):

$$
d_{g}\left(p_{w i}, p_{w j}\right):=\rho \cos ^{-1}\left(\frac{p_{w i}^{\mathrm{T}} p_{w j}}{\rho^{2}}\right) .
$$

Then, substituting (2) into (8) can rewrite $d_{g}\left(p_{w i}, p_{w j}\right)$ as

$$
d_{g}\left(e^{\hat{\xi} \theta_{i j}}\right)=\rho \cos ^{-1}\left(e_{3}^{\mathrm{T}} e^{\hat{\xi} \theta_{i j}} e_{3}\right),
$$

that is, the geodesic distance $d_{g}$ is formed by the relative attitude $e^{\hat{\xi} \theta_{i j}}$.

Remark 2 The geodesic distance $d_{g}$ is defined by using $\cos ^{-1}(\cdot)$, but its argument $e_{3}^{\mathrm{T}} e^{\hat{\xi} \theta_{i j}} e_{3}$ has a value within the region $[-1,1]$. Therefore, the geodesic distance $d_{g}$ is always well defined as the shortest arc length on the spherical surface. The situation that two rigid bodies exist perfectly at the opposite positions is the special case because we have the infinite number of arcs to determine $d_{g}$. However, such an undesired situation can be avoided by appropriately setting distances for collision avoidance (discussed in Section IV-B].

From the geodesic distance definition (9), we define collisions between rigid bodies and collision avoidance for a rigid body network on a sphere as follows (see Fig. 4 (b)):

Definition 2 The collision between rigid body $i$ and rigid body $j$ occurs when $d_{g}\left(e^{\hat{\xi} \theta_{i j}}\right)<D_{c}$, where $D_{c}>0$ is their geodesic diameter. Then, collision avoidance is achieved for a rigid body network on a sphere if

$$
d_{g}\left(e^{\hat{\xi} \theta_{i j}}(t)\right) \geq D_{c} \forall i, j \in \mathcal{V}(i \neq j), t \geq 0 .
$$

In this formulation, the objective of this paper is to design conic $\mathrm{CBF}$, derive conditions, and propose a distributed method for rigid body $i \in \mathcal{V}$ in order to achieve the collision avoidance (10) for a rigid body network on a sphere.

\section{B. Distributed Collision Avoidance on a Sphere}

Define the following safe set $C$ for a rigid body network on a sphere:

$$
C:=\left\{e^{\hat{\xi} \theta_{w i}}, i \in \mathcal{V} \mid d_{g}\left(e^{\hat{\xi} \theta_{i j}}\right) \geq D_{c} \forall i, j \in \mathcal{V}(i \neq j)\right\}
$$

Then, the collision avoidance 10 is equivalent to the forward invariance of the safe set $C$. Let us now assume that the collision distance $D_{c}$ satisfies $D_{c}<(\rho \pi) / 2$. This assumption is reasonable since this inequality means that the geodesic diameter of each rigid body is less than one fourth of the circumference of the sphere, i.e., the size of each rigid body is not too large compared with that of the sphere.

We note that each rigid body is required to take collision avoidance behaviors only when it approaches other rigid bodies. Besides the graph $\mathcal{G}$ for a given motion coordination strategy, therefore, we introduce another geodesic distance based undirected graph $\mathcal{G}^{\prime}=\left(\mathcal{V}, \mathcal{E}^{\prime}\right), \quad \mathcal{E}^{\prime}:=\{(i, j) \in$ $\left.\mathcal{V}(i \neq j) \mid d_{g}\left(e^{\hat{\xi} \theta_{i j}}\right) \leq D_{a} \forall i, j \in \mathcal{V}\right\}$. Here, $D_{a}>D_{c}$ is the geodesic distance within which rigid bodies take account of collision avoidance behaviors. According to $\mathcal{G}^{\prime}$, we also define a new neighbor set of rigid body $i$ for the collision avoidance (10), called distance neighbors, as $\mathcal{N}_{d, i}:=\left\{j \in \mathcal{V} \mid(j, i) \in \mathcal{E}^{\prime}\right\}$. Notice now that by employing the reasonable assumption $D_{a}<(\rho \pi) / 2$, we can avoid the undesired special case stated in Remark 2 i.e., the existence of a distance neighbor perfectly at the opposite position on the sphere, in the collision avoidance process.

Let us define the conic $\mathrm{CBF}$ candidates as

$$
h_{i j}:=-e_{3}^{\mathrm{T}} e^{\hat{\xi} \theta_{i j}} e_{3}+\cos \left(\frac{D_{c}}{\rho}\right) \in \mathbb{R}
$$

for $i, j \in \mathcal{V}$, where we take $\cos (\cdot)$ for the geodesic distance. Then, by rewriting (11) as

$$
C=\left\{e^{\hat{\xi} \theta_{w i}}, i \in \mathcal{V} \mid h_{i j} \geq 0 \forall i, j \in \mathcal{V}(i \neq j)\right\},
$$

the forward invariance of the safe set $C$ for a rigid body network on a sphere is analogous to collision-free motions. We now have the following theorem showing the achievement of the collision avoidance (10):

Theorem 2 Suppose that collisions do not occur in a rigid body network on a sphere at the initial time, i.e., $\left\{e^{\hat{\xi} \theta_{w i}}(0)\right\}_{i \in \mathcal{V}} \in C$. Then, any Lipschitz continuous controllers $\omega_{i}, i \in \mathcal{V}$ satisfying

$$
\begin{array}{r}
e_{3}^{\mathrm{T}} e^{-\hat{\xi} \theta_{i j}} \hat{e}_{3} \omega_{i} \geq k\left(e_{3}^{\mathrm{T}} e^{\hat{\xi} \theta_{i j}} e_{3}-\cos \left(\frac{D_{c}}{\rho}\right)\right) \\
\forall j \in \mathcal{N}_{d, i}, k>0
\end{array}
$$

will render the safe set $C$ forward invariant.

Proof: The following condition is first derived from $\dot{h}_{i j}\left(e^{\hat{\xi} \theta_{i j}}\right)+2 k h_{i j}\left(e^{\hat{\xi} \theta_{i j}}\right) \geq 0$ for each rigid body $i \in \mathcal{V}$ :

$$
\begin{aligned}
e_{3}^{\mathrm{T}} e^{-\hat{\xi} \theta_{i j}} & \hat{e}_{3} \omega_{i}+e_{3}^{\mathrm{T}} e^{\hat{\xi} \theta_{i j}} \hat{e}_{3} \omega_{j} \\
& \geq 2 k\left(e_{3}^{\mathrm{T}} e^{\hat{\xi} \theta_{i j}} e_{3}-\cos \left(\frac{D_{c}}{\rho}\right)\right) \forall j \in \mathcal{N}_{d, i} .
\end{aligned}
$$

Here, $\alpha(h)=2 k h, k>0$ is employed as an extended class $\mathcal{K}$ function, and only the distance neighbors $j \in \mathcal{N}_{d, i}$ are considered because we have $h_{i j}\left(e^{\hat{\xi} \theta_{i j}}\right)>0$ for any $j \in$ $\mathcal{V} \backslash \mathcal{N}_{d, i}(j \neq i)$ from $D_{a}>D_{c}$.

The condition (13) for each rigid body $i$ is not distributed since it requires input information of distance neighbors, i.e., $\omega_{j}, j \in \mathcal{N}_{d, i}$. We thus employ the distributed condition (12) 
to satisfy (13). Then, because $j \in \mathcal{N}_{d, i} \Leftrightarrow i \in \mathcal{N}_{d, j}$ and $e_{3}^{\mathrm{T}} e^{\hat{\xi} \theta_{i j}} e_{3}=e_{3}^{\mathrm{T}} e^{\hat{\xi} \theta_{j i}} e_{3}$ hold, the satisfaction of (12) for all $i \in \mathcal{V}$ guarantees (13) for all $i \in \mathcal{V}$. Here, considering (12) can be regarded as sharing (13) equally $3^{3}$ between rigid body $i$ and rigid body $j$. Theorem 1 can be thus applied.

Remark 3 The condition (12) is based only on information about distance neighbors $j \in \mathcal{N}_{d, i}$, i.e., distributed. Moreover, (12) is based only on relative attitude information $e^{\hat{\xi} \theta_{i j}}$ viewed from $\Sigma_{i}$ since $e^{-\hat{\xi} \theta_{i j}}=\left(e^{\hat{\xi} \theta_{i j}}\right)^{\mathrm{T}}$ holds.

\section{Collision-Free Motion Coordination on a Sphere}

Based on Theorem 2 we propose, for rigid body $i \in \mathcal{V}$ in a rigid body network on a sphere, the following control input provided by the solution of the quadratic program to guarantee the collision avoidance (10):

$$
\omega_{i}^{*}=\arg \min _{\omega_{i} \in \mathbb{R}^{3}}\left\|\omega_{i}-\omega_{\text {nom }, i}\right\|^{2} \text { s.t. (12). }
$$

Here, $\omega_{n o m, i} \in \mathbb{R}^{3}, i \in \mathcal{V}$ are the nominal control inputs to achieve a given motion coordination strategy. The optimization in (14) implies that each rigid body tries to achieve the given motion coordination task as much as possible while guaranteeing the collision avoidance (10).

Remark 4 The optimization in (14) is always feasible in the safe set $C$ since it has at least one feasible solution $\omega_{i}=0$. From the same reason, we can easily impose an additional input saturation constraint, e.g., $\left\|\omega_{i}\right\| \leq \omega_{\max }$ for some $\omega_{\max }>0$. In this case, we can replace $\omega_{i} \in \mathbb{R}^{3}$ in (14) with $\omega_{i} \in \Omega, \Omega=\left\{\omega \in \mathbb{R}^{3} \mid\|\omega\| \leq \omega_{\max }\right\}$.

Any motion coordination strategy can be applied as $\omega_{\text {nom }, i}$ in (14). In the simulation verification presented in Section $\nabla$ we apply the following attitude synchronization law [17]:

$$
\omega_{n o m, i}=k_{c} \sum_{j \in \mathcal{N}_{i}} \operatorname{sk}\left(e^{\hat{\xi} \theta_{i j}}\right)^{\vee} .
$$

Here, $k_{c}>0$ is the controller gain, and $\operatorname{sk}\left(e^{\hat{\xi} \theta}\right):=$ $(1 / 2)\left(e^{\hat{\xi} \theta}-e^{-\hat{\xi} \theta}\right)=\hat{\xi} \theta \in s o(3)$. Then, it is shown in [17] that if the initial attitudes of a rigid body network satisfy $\left|\theta_{i j}(0)\right|<\pi \forall i, j \in \mathcal{V}$ and the interconnection topology $\mathcal{G}$ is fixed and strongly connected, the control input $\omega_{i}=\omega_{n o m, i}$ given by (15) achieves the attitude synchronization defined as follows:

$$
\lim _{t \rightarrow \infty}\left\|e^{\hat{\xi} \theta_{w i}}(t)-e^{\hat{\xi} \theta_{w j}}(t)\right\|_{F}=0 \forall i, j \in \mathcal{V} .
$$

Here, $\|\cdot\|_{F}$ is the Frobenius norm.

In the case of a rigid body network on a sphere under the spherical constraint (2), the attitude synchronization (16) also implies the position synchronization defined as

$$
\lim _{t \rightarrow \infty}\left\|p_{w i}(t)-p_{w j}(t)\right\|=0 \forall i, j \in \mathcal{V} .
$$

Then, by applying the control input (14) with the nominal input (15), we can expect the achievement of a flockinglike behavior: cohesion; alignment; and separation [32], on

\footnotetext{
${ }^{3}$ As generalization of the equally sharing, we can also introduce weights $w_{i j} \in \mathbb{R},(j, i) \in \mathcal{E}^{\prime}$ satisfying $w_{i j}+w_{j i}=1$ to share the condition [13.
}

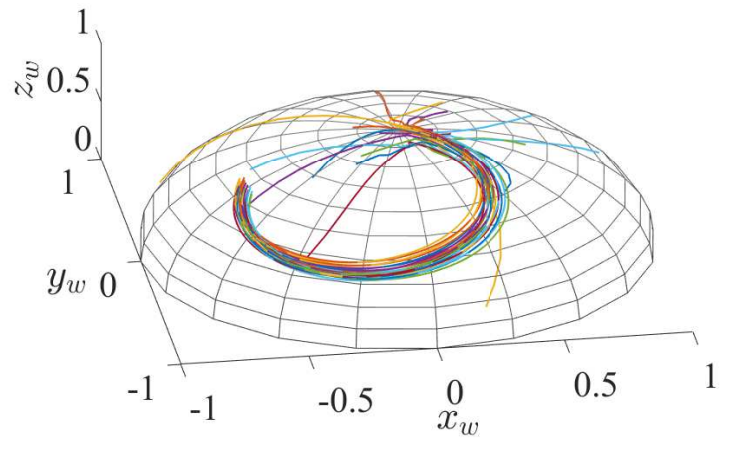

(a) Position trajectories in $\Sigma_{w}$.

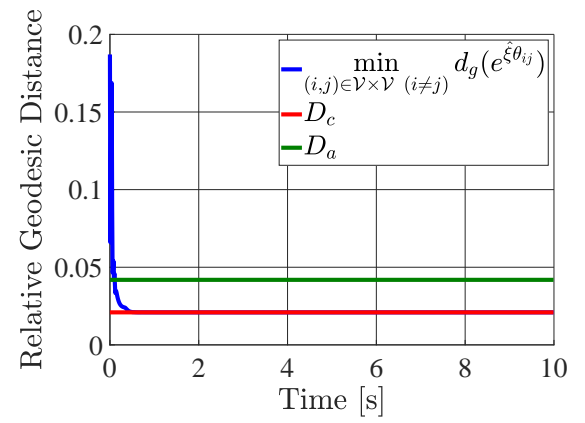

(b) Time response of minimum geodesic distance.

Fig. 5. Simulation results. (a): The rigid body network achieves the cohesion and alignment behaviors on the sphere. (b): The minimum geodesic distance never becomes less than the collision distance $D_{c}$, i.e., the collision-free motion coordination (separation behavior) is achieved.

a sphere. Here, the final control input of rigid body $i \in \mathcal{V}$ is distributed and based only on relative attitudes with respect to $j \in \mathcal{N}_{i} \cup \mathcal{N}_{d, i}$, which can be implemented in a distributed manner using onboard sensors, e.g., vision or infrared, without any other communication or global information.

\section{SIMULATION}

Simulation is conducted to demonstrate the validity of the proposed collision-free motion coordination method (14), (15). Here, we slightly modify each control input by adding the common rotational body velocity $\omega_{c}=\left[\begin{array}{lll}0.1 & 0.2 & -0.4\end{array}\right] \in$ $\mathbb{R}^{3}$ to make it easy to see the final configuration of a rigid body network on a sphere. Even with this modification, the relative attitude dynamics are not changed, i.e., the same behavior in the sense of the relative states can be seen.

Consider a rigid body network on a sphere with 20 rigid bodies and strongly connected interconnection topology $\mathcal{G}$. The simulation parameters are set as $\rho=1$, (i.e., $D_{c}=\theta_{c}$ ), $\theta_{c}=\pi / 150, D_{a}=2 D_{c}, k=1$, and $k_{c}=5$. The initial positions are set so that each rigid body $i \in \mathcal{V}$ exists in the upper half of the sphere in the $z$-axis direction of $\Sigma_{w}$.

The simulation results are shown in Fig. 5] Fig. 5(a) illustrates the position trajectories of the rigid body network on a sphere in $\Sigma_{w}$, which demonstrates the proposed control method achieves the cohesion and alignment behaviors. The collision avoidance (10) can be confirmed by Fig 5 b) depicting the time responses of the minimum geodesic distance between the rigid body pairs $(i, j) \in \mathcal{V} \times \mathcal{V}(i \neq j)$. 


\section{CONCLUSIONS}

This paper presented a distributed collision avoidance control method for a group of multiple rigid bodies on a sphere. Based on the fact that the rigid body motion constrained to a spherical surface is equivalent to attitude motion on $S O(3)$, the collision avoidance law is derived with conic control barrier functions on $S O(3)$ that can handle geodesic distance constraints on a spherical surface. In the proposed method, each rigid body chooses its control input by solving a distributed optimization problem to achieve a given motion coordination strategy while satisfying constraints for collision avoidance derived by the conic control barrier functions. The validity of the proposed approach was demonstrated via simulation.

\section{APPENDIX}

\section{A. Local Lipschitz Continuity of Attitude Dynamics (3b)}

It is well known that according to the subspace $\mathcal{S} \subset$ $S O(3)$, the attitude dynamics (3b) can be rewritten in vector form using the Euler angles, quaternions, Cayley parameters, etc. [26]-[28]. We here introduce the XYZ Euler angle case.

Consider the XYZ Euler angle representation: $\left(\phi_{i}, \psi_{i}, \eta_{i}\right)$, e.g., in the region $\phi_{i}, \psi_{i}, \eta_{i} \in(-\pi / 2, \pi / 2)$. Then, the rotation matrix $e^{\hat{\xi} \theta_{w i}}$ can be uniquely given as $e^{\hat{\xi} \theta_{w i}}=$ $R_{x}\left(\phi_{i}\right) R_{y}\left(\psi_{i}\right) R_{z}\left(\eta_{i}\right)$ in the subspace $\mathcal{S}$ according to the region of the Euler parameters. Here, $R_{x}, R_{y}, R_{z} \in S O(3)$ are respectively the basis rotation matrices with respect to $x$-, $y$-, and $z$-axes [25]. Then, with $\zeta_{i}:=\left[\phi_{i} \psi_{i} \eta_{i}\right]^{\mathrm{T}} \in \mathbb{R}^{3}$, the attitude dynamics (3b) are analogous to

$$
\dot{\zeta}_{i}=\left[\begin{array}{ccc}
\frac{\cos \eta_{i}}{\cos \psi_{i}} & -\frac{\sin \eta_{i}}{\cos \psi_{i}} & 0 \\
\sin \eta_{i} & \cos \eta_{i} & 0 \\
-\cos \eta_{i} \tan \psi_{i} & \sin \eta_{i} \tan \psi_{i} & 1
\end{array}\right] \omega_{i}=: g\left(\zeta_{i}\right) \omega_{i}
$$

Since $g\left(\zeta_{i}\right)$ consists of smooth trigonometric functions, the attitude dynamics are locally Lipschitz continuous on the subspace $\mathcal{S}$.

\section{REFERENCES}

[1] F. Bullo, J. Cortés, and S. Martínez, Distributed Control of Robotic Networks: A Mathematical Approach to Motion Coordination Algorithms. Princeton, NJ, USA: Princeton Univ. Press, 2009.

[2] M. Mesbahi and M. Egerstedt, Graph Theoretic Methods for Multiagent Networks. Princeton, NJ, USA: Princeton University Press, 2010.

[3] W. Ren and R. W. Beard, Distributed Consensus in Multi-vehicle Cooperative Control: Theory and Applications. London, U.K.: SpringerVerlag, 2008.

[4] T. Ibuki, S. Wilson, J. Yamauchi, M. Fujita, and M. Egerstedt, "Optimization-based distributed flocking control for multiple rigid bodies," IEEE Robot. Autom. Lett., vol. 5, no. 2, pp. 1891-1899, Apr. 2020.

[5] S. Martínez, J. Cortés, and F. Bullo, "Motion coordination with distributed information," IEEE Control Syst. Mag., vol. 27, no. 4, pp. 75-88, Aug. 2007.

[6] V. Muralidharan, A. D. Mahindrakar, and A. Saradagi, "Control of a driftless bilinear vector field on $n$-sphere," IEEE Trans. Autom. Control, vol. 64, no. 8, pp. 3226-3228, Aug. 2019.

[7] J. Markdahl, J. Thunberg, and J. Goncalves, "Almost global consensus on the $n$-sphere," IEEE Trans. Autom. Control, vol. 63, no. 6, pp. 1664-1675, Jun. 2018.

[8] S. Al-Abri and F. Zhang, "Consensus on a sphere for a 3-dimensional speeding up and slowing down strategy," in Proc. 56th IEEE Conf. Decis. Control, 2017, pp. 1503-1508.
[9] C. Lageman and Z. Sun, "Consensus on spheres: Convergence analysis and perturbation theory," in Proc. 55th IEEE Conf. Decis. Control, 2016, pp. 19-24.

[10] W. Li, "Collective motion of swarming agents evolving on a sphere manifold: A fundamental framework and characterization," Sci. Rep., vol. 5, 2015, Art. no. 13603

[11] W. Li and M. W. Spong, "Unified cooperative control for multiple agents on a sphere for different spherical patterns," IEEE Trans. Autom. Control, vol. 59, no. 5, pp. 1283-1289, May 2014.

[12] S. Hernandez and D. A. Paley, "Three-dimensional motion coordination in a spatiotemporal flowfield," IEEE Trans. Autom. Control, vol. 55, no. 12 , pp. 2805-2810, Dec. 2010.

[13] R. Olfati-Saber, "Swarms on sphere, A programmable swarm with synchronous behaviors like oscillator networks," in Proc. 45th IEEE Conf. Decis. Control, 2006, pp. 5060-5066.

[14] T. Lee, "Geometric control of multiple quadrotor UAVs transporting a cable suspended rigid body," in Proc. 53rd IEEE Conf. Decis. Control, 2014, pp. 6155-6160.

[15] G. Wu and K. Sreenath, "Geometric control of multiple quadrotors transporting a rigid-body load," in Proc. 53rd IEEE Conf. Decis. Control, 2014, pp. 6141-6148.

[16] C. K. Verginis and D. V. Dimarogonas, "Closed-form barrier functions for multi-agent ellipsoidal systems with uncertain Lagrangian dynamics," IEEE Control Syst. Lett., vol. 3, no. 3, pp. 727-732, Jul. 2019.

[17] T. Hatanaka, N. Chopra, M. Fujita, and M. W. Spong, Passivity-based Control and Estimation in Networked Robotics. Cham, Switzerland: Springer, 2015.

[18] L. Sabattini, C. Secchi, and N. Chopra, "Decentralized connectivity maintenance for networked Lagrangian dynamical systems with collision avoidance," Asian J. Control, vol. 17, no. 1, pp. 111-123, Jan. 2015.

[19] D. M. Stipanović, P. F. Hokayem, M. W. Spong, and D. D. Šiljak, "Cooperative avoidance control for multiagent systems," J. Dyn. Syst., Meas., Control, vol. 129, no. 5, pp. 699-707, Apr. 2007.

[20] D. V. Dimarogonas, S. G. Loizou, K. J. Kyriakopoulos, and M. M. Zavlanos, "A feedback stabilization and collision avoidance scheme for multiple independent non-point agents," Automatica, vol. 42, pp. 229-243, Feb. 2006.

[21] A. D. Ames, X. Xu, J. W. Grizzle, and P. Tabuada, "Control barrier function based quadratic programs for safety critical systems," IEEE Trans. Autom. Control, vol. 62, no. 8, pp. 3861-3876, Aug. 2017.

[22] S. Kolathaya and A. D. Ames, "Input-to-state safety with control barrier functions," IEEE Control Syst. Lett., vol. 3, no. 1, pp. 108113, Jan. 2019.

[23] L. Wang, A. D. Ames, and M. Egerstedt, "Safety barrier certificates for collisions-free multirobot systems," IEEE Trans. Robot., vol. 33, no. 3, pp. 661-674, Jun. 2017.

[24] D. Panagou, D. M. Stipanović, and P. G. Voulgaris, "Distributed coordination control for multi-robot networks using Lyapunov-like barrier functions," IEEE Trans. Autom. Control, vol. 61, no. 3, Mar. 2016.

[25] R. M. Murray, Z. Li, and S. S. Sastry, A Mathematical Introduction to Robotic Manipulation. Boca Raton, FL, USA: CRC Press, 1994.

[26] N. A. Chaturvedi, A. K. Sanyal, and N. H. McClamroch, "Rigid-body attitude control," IEEE Control Syst. Mag., vol. 31, no. 3, pp. 30-51, Jun. 2011

[27] H. Schaub, P. Tsiotras, and J. L. Junkins "Principal rotation representations of proper $N \times N$ orthogonal matrices," Int. J. Eng. Sci., vol. 33, no. 15, pp. 2277-2295, Dec. 1995.

[28] D. Q. Huynh, "Metrics for 3D rotations: Comparison and analysis," J. Math. Imaging Vis., vol. 35, pp. 155-164, Jun. 2009.

[29] A. Weiss, F. Leve, M. Baldwin, J. R. Forbes, and I. Kolmanovsky, "Spacecraft constrained attitude control using positively invariant constraint admissible sets on $S O(3) \times \mathbb{R}^{3}$," in Proc. Amer. Control Conf., 2014, pp. 4955-4960.

[30] S. Kulumani and T. Lee, "Constrained geometric attitude control on SO(3)," Int. J. Control, Autom. Syst., vol. 15, no. 6, pp. 2796-2809, Dec. 2017.

[31] S. Nakano, T. W. Nguyen, E. Garone, T. Ibuki, and M. Sampei, "Attitude constrained control on $S O(3)$ : An explicit reference governor approach," in Prof. 57th IEEE Conf. Decis. Control, 2018, pp. 18331838.

[32] C. W. Reynolds, "Flocks, herds and schools: A distributed behavioral model," Comput. Graph., vol. 21, no. 4, pp. 25-34, Jul. 1987. 